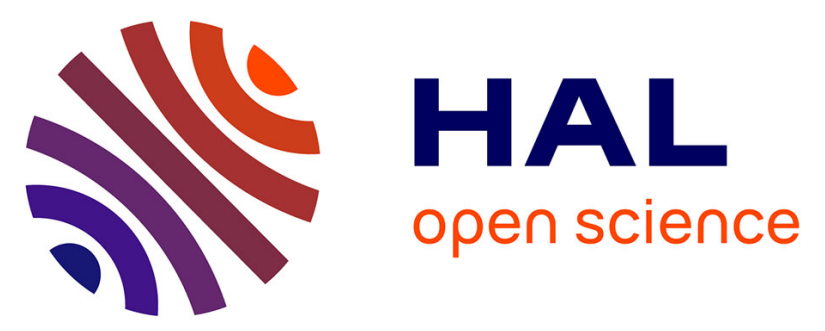

\title{
Contrast-enhanced CT texture parameters as predictive markers of high-risk urodynamic features in adult patients with spina bifida
}

Zine-Eddine Khene, Claire Richard, Juliette Hascoet, Anis Gasmi, Anna Goujon, Charlène Brochard, Magali Jezequel, Quentin Alimi, Laurent Siproudhis, Guillaume Bouguen, et al.

\section{To cite this version:}

Zine-Eddine Khene, Claire Richard, Juliette Hascoet, Anis Gasmi, Anna Goujon, et al.. Contrastenhanced CT texture parameters as predictive markers of high-risk urodynamic features in adult patients with spina bifida. Urology, 2019, 134, pp.84-89. 10.1016/j.urology.2019.09.023 . hal-02363655

\section{HAL Id: hal-02363655}

\section{https://hal-univ-rennes1.archives-ouvertes.fr/hal-02363655}

Submitted on 9 Dec 2019

HAL is a multi-disciplinary open access archive for the deposit and dissemination of scientific research documents, whether they are published or not. The documents may come from teaching and research institutions in France or abroad, or from public or private research centers.
L'archive ouverte pluridisciplinaire HAL, est destinée au dépôt et à la diffusion de documents scientifiques de niveau recherche, publiés ou non, émanant des établissements d'enseignement et de recherche français ou étrangers, des laboratoires publics ou privés. 


\section{Contrast-enhanced CT texture parameters as predictive markers of high-risk urodynamic features in adult patients with spina bifida}

Zine-eddine Khene ${ }^{1}$, Claire Richard ${ }^{1}$, Juliette Hascoet ${ }^{1,2,3}$, Anis Gasmi ${ }^{1}$, Anna Goujon

${ }^{1}$, Charlène Brochard ${ }^{2,3,4}$, Magali Jezequel ${ }^{2}$, Quentin Alimi ${ }^{1,2}$, Laurent Siproudhis ${ }^{2,3,4}$, Guillaume Bouguen ${ }^{3,4}$, Jacques Kerdraon ${ }^{2,5}$, Andrea Manunta ${ }^{1,2}$, Xavier Gamé ${ }^{10}$, Romain Mathieu ${ }^{1}$, Benoit Peyronnet ${ }^{1,2,3}$

1: Service d'urologie, CHU Rennes, 35000 Rennes, France

2: Centre de référence spina bifida, CHU Rennes, 35000 Rennes, France

3: Equipe thématique INPHY CIC 1414 et INSERM UMR 991, CHU Rennes, 35000 Rennes, France

4 : Service de Gastro-Entérologie, CHU Rennes, 35000 Rennes, France

5: Centre de rééducation de Kerpape, 56270 Ploemeur, France

6: Département d'Urologie, Transplantation Rénale et Andrologie, CHU Rangueil, 33000 Toulouse, France

\section{Corresponding author}

Benoit Peyronnet

Service d'Urologie, Hopital Pontchaillou,

2 rue Henri Le Guilloux, 35000 Rennes, France

mail: peyronnetbenoit@hotmail.fr

Tel: +33299284321

Keywords (MeSH): urinary bladder; spina bifida; neurogenic; urodynamic; Computed tomography; texture analysis

Word count: 2569

\section{Conflicts of interest}

The authors have nothing to disclose 


\section{Abstract}

\section{$\underline{\text { Objective }}$}

to investigate computed tomography (CT) texture analysis of the bladder wall as a predictor of urodynamics findings in adult patient with spina bifida.

\section{Methods}

A single-center prospective trial was conducted from March 2015 to March 2017 including all consecutive adult spina bifida patients seen for urodynamic testing. A contrast-enhanced abdominal CT was systematically performed in all patients during the same visit. Texture features of the bladder wall related to the gray-level histogram and gray-level co-occurrence were evaluated on CT images. Multivariate analysis was performed to identify independent predictors of poor bladder compliance (PBC) and detrusor overactivity (DO) among clinical and texture parameters.

\section{$\underline{\text { Results }}$}

Fourty patients were included. The Lasso penalized logistic regression analysis identified two texture parameters as potential predictors of poor bladder compliance: Skewness (coefficient weight, -1.81) and S.1.1.SumVarnc (coefficient weight, -3.52). Multivariate Logistic regression analysis confirmed skewness (OR $(\mathrm{Cl}$ 95\%) $=0.40$ $(0.14,0.97), p=0.04)$ as an independent predictor of PBC. The Lasso penalized logistic regression analysis identified one texture parameters as potential predictor of DO: Kurtosis (coefficient weight, -3.52). which was confirmed in multivariate logistic regression analysis $(\mathrm{OR}(\mathrm{Cl} 95 \%)=1.12(1.01,1.55), \mathrm{p}=0.02$.

\section{Conclusion}

Our findings demonstrate that CT texture analysis of the bladder wall might be an interesting tool to identify spina bifida patients with high risk urodynamic features. 


\section{Introduction}

Spina bifida is a birth defect originally defined as an incomplete closure of the neural tube in the caudal region resulting in protrusion of part or all of the content of the spinal canal through this dorsal defect [1]. Spina bifida is the most common congenital cause of neurogenic bladder with an incidence of 1/10,000 births in developed countries [1-2]. Neurogenic lower urinary tract dysfunction (NLUTD) is present in more than $90 \%$ of spina bifida patients and carries a high-risk of upper urinary tract damage [3]. For that reason, most guidelines recommend to repeat urodynamics over follow-up to monitor bladder pressure and tailor therapeutic management [4]. However, repeat urodynamics cause a severe burden to spina bifida patients due to its invasiveness and are costly for the healthcare systems. Several non-invasive alternatives to urodynamics have been evaluated over the past decade [5] but, so far, none has been deemed reliable enough to be implemented in daily practice. In the last decade, there has been an increasing interest in the analysis of quantitative information from medical images to improve the performance and usefulness of imaging. Textural analysis relies on objective computerassisted measurements and explore the spatial variations of signal intensity in imaging [6]. Numerous published articles have shown the ability of texture analysis algorithms to extract information from various imaging modalities in several fields [7]. We aimed to investigate computed tomography (CT) texture analysis of the bladder wall as a predictor of urodynamics findings in adult patient with spina bifida.

\section{Materials and Methods:}

\section{Study design}

From March, 2015 to March, 2017 all adult spina bifida patients seen at the national referral center for spina bifida were offered a routine urological evaluation whether they had urological complaints or not. All consecutive patients undergoing urodynamics for the assessment of their lower urinary tract function were enrolled in a prospective study (ClinicalTrials.gov Identifier: NCT02852317). All study materials were reviewed and approved by the local Independent Ethics Committee, and all patients provided written informed consent. Patients were excluded if they had an ongoing symptomatic urinary 
tract infection, or a history of augmentation cystoplasty or urinary tract malignancy. The following variables were collected for each patient: demographics, past medical history, physical examination, bladder management (self-catheterization vs. spontaneous voiding) bladder diary data, urine culture, types of spinal dysraphism (open vs. closed), ongoing and previous bladder medications (anticholinergics, beta-3 agonists,...), and history of intradetrusor botulinum toxin injections. The type of spinal dysraphism (open vs. closed) was defined based on previous spine imaging and operative reports and on a Magnetic Resonance Imaging (MRI) of the spine performed at the time of the first visit for every patients. The sensory-motor level of neurological impairment was categorized as sacral, lumbar or thoracic.

\section{Urological assessment}

At the first visit, lower urinary tract function was systematically assessed regardless of the presence or not of lower urinary tract symptoms (LUTS). Each single patient during his/her first visit to the center was seen in clinics by a urologist. The urologist's assessment included: history of past urological surgery, bladder management (cleanintermittent catheterization (CIC) vs. spontaneous voiding), urinary incontinence defined as any urine leakage reported by the patient at least once a week or evidenced during clinical examination or by the use of any pads, oral anticholinergics intake (yes vs. no). All patients underwent a morphological evaluation of the urinary tract using abdominal computerized tomography (CT, see details below) as part of their initial urological assessment.

\section{Urodynamic evaluation}

All patients seen at the French referral center for spina bifida were offered a urodynamic study at their first visit even though they came to the center for a non-urological issue and were completely asymptomatic from the urological standpoint. Standard filling cystometry using saline was done with patients in the supine position using a 7Fr to $10 \mathrm{Fr}$ double lumen urethral cystometry catheter and a rectal balloon catheter according to the International Continence Society (ICS) standards [8]. Anticholinergic therapy was discontinued at least one week before urodynamic evaluation. Filling cystometry was 
then performed at a rate of $20 \mathrm{~mL} / \mathrm{min}$ with saline. Neurogenic detrusor overactivity (NDO) was defined in accordance with the recent standardization of terminology report by the ICS as a urodynamic observation characterized by involuntary detrusor contractions during the filling phase which may be spontaneous or provoked in the setting of a clinically relevant neurologic disease [9]. The following urodynamics data, defined according to the ICS guidelines [8], were also collected: cystometric capacity (CC, $\mathrm{ml})$, volume at first sensation of bladder filling $(\mathrm{ml})$, volume at first uninhibited detrusor contraction if any $(\mathrm{ml})$, bladder compliance calculated manually from the traces $(\mathrm{ml} / \mathrm{cmH} 2 \mathrm{O})$, maximum detrusor pressure (MDP, $\mathrm{cmH} 2 \mathrm{O})$. Poor bladder compliance was defined as bladder compliance $<20 \mathrm{ml} / \mathrm{cmH} 2 \mathrm{O}$ according to the ICS standardization report [8].

Quantitative computed tomography Texture analysis of bladder wall CT technique All patients underwent contrast-enhanced CT. Patients were asked to present with a full bladder for the CT. To obtain three representative images per patient, regions of interest (ROIs) were defined in the coronal plane at three levels through the craniocaudal dimension of the bladder corresponding to approximately the upper third, center and lower third of the organ (figure 1). The area and position of the ROI for each level was kept constant.

\section{CT Texture Analysis}

Each image was collected and stored in the original digital imaging and communication in medicine (DICOM) format. Images were anonymized. These images were then transferred to an independent workstation for lesion segmentation by using ImageJ software (National Institutes of Health, http://rsbweb. nih.gov/). For each image a ROI was manually drawn slightly on enhanced image. Prior to analysis, CT image intensities were normalized between $\mu+/-3 s$, where $\mu$ was the mean value of gray levels inside the region of interest and $s$ was the standard deviation. This normalization procedure has been shown to minimize inter-scanner effects in MRI texture analysis and is presumed to also reduce inter-scanner effects in other modalities such as CT (figure 2). Texture 
features related to the gray-level histogram and gray-level co-occurrence (GLCM), were evaluated using MaZda, version 4.6 (P.M. Szczypiński, Institute of Electronics, Technical University of Lodz, Poland).

Statistical analysis

We restricted our analysis to patient-level means for each feature and for each set of contours. Quantitative variables were described as means with standard deviation or medians with minimum-maximum and categorical variables as percentages. Multivariate analysis was performed to identify independent predictors of poor bladder compliance and detrusor overactivity among clinical and texture parameters. To take into consideration the correlation between the estimates of each texture parameter and the small number of events, a multivariate L1 (least absolute shrinkage and selection operator-Lasso) penalized logistic regression model was built in order to select texture parameters. The regularization parameter was determined by using tenfold crossvalidation. The Lasso method allows variable selection by shrinking down to zero coefficient weights for variables non-related to the outcome. Variables with non-zero coefficients were selected as potential predictors of outcome and integrated into a multivariable logistic regression analysis, in order to estimate associated hazard ratios (HR) and their 95\% confidence intervals (Cl 95\%). For each texture parameter predictor of outcome, an additional analysis was performed to determine the optimal threshold dividing out patients with good and poor prognosis, based on the threshold with the highest Youden index using non-parametric test. All statistical analyzes were performed using $R$ software ( $R$ Foundation for Statistical Computing, Vienna, Austria) and STATA 13.0 (College Station, TX).

\section{Results}

\section{Patient characteristics}

Forty patients were enrolled over the study period. The patients' characteristics are summarized in supplementary table 1 . The mean patients' age was 37.7 years and there 
was $52.5 \%$ and $48.5 \%$ of male and female patients respectively. The majority of patients were self-catheterizing (70\%) and the predominant neurological level was lumbar $(79 \%)$. The mean maximum voided volume on the voiding diary was $419.7 \mathrm{ml}$. Eleven patients had signs of upper urinary tract damage on imaging (27.5\%): hydronephrosis in eight patients $(20 \%)$, renal scaring in two patients $(5 \%)$ and renal atrophy in two patients $(5 \%)$. The urodynamic findings are shown in supplementary table 2 . The mean CC, MDP and volume at first sensation of bladder filling were $419.7 \mathrm{~mL}, 24.4 \mathrm{cmH} 2 \mathrm{O}$ and $225.7 \mathrm{~mL}$ respectively. There were 15 patients with poor bladder compliance (37.5\%) and 15 patients with DO (37.5\%).

Poor bladder compliance analysis

The Lasso penalized logistic regression analysis identified two texture parameters as potential predictors of poor bladder compliance: Skewness (coefficient weight, -1.81) and S.1.1.SumVarnc (coefficient weight, -3.52). Two clinical parameters also highlighted non-zero coefficient weights: age (coefficient weight, -1.43), and type of spinal dysraphism (coefficient weight 3.55). Multivariate Logistic regression analysis confirmed skewness $(\mathrm{OR}(\mathrm{Cl} 95 \%)=0.40(0.14,0.97), \mathrm{p}=0.04)$, and age $(\mathrm{HR}(\mathrm{Cl} 95 \%)=0.96$ $(0.92,0.995), p=0.02)$ as independent predictors of poor bladder compliance (table 1$)$. When dichotomized at the optimal threshold, skewness under -0.417 was significantly associated with high risk of poor bladder compliance $(p=0.02790)$.

\section{Detrusor overactivity}

The Lasso penalized logistic regression analysis identified one texture parameters as potential predictor of detrusor overactivity: Kurtosis (coefficient weight, -3.52). Four clinical parameters also highlighted non-zero coefficient weights: age (coefficient weight, -1.42), type of spinal dysraphism (coefficient weight 3.57), neurological level (coefficient weight 3.57) and male gender (coefficient weight 1.43). Multivariate Logistic regression analysis showed that only kurtosis $(\mathrm{OR}(\mathrm{Cl} 95 \%)=1.12(1.01,1.55), \mathrm{p}=0.02)$ was an independent predictor of detrusor overactivity (table 2). When dichotomized at the 
optimal threshold, kurtosis under 1.48 was significantly associated with high risk of detrusor overactivity $(p=0.0212)$

\section{Discussion}

Spina bifida is the most common congenital cause of NLUTD [2]. Spina bifida patients are subject to severe NLUTD which often have very specific features with high prevalence of acontractile detrusor, poor bladder compliance and intrinsic sphincter deficiency [3]. Until now, initial assessment and follow-up of spina bifida NLUTD has been relying heavily upon urodynamics as being the only exam able to properly identify and characterize NLUTD, with some literature showing correlations with the risk of upper urinary tract deterioration [10-11]. However, urodynamics have its cons including the invasiveness of urethral catheterization, discomfort of the exam for the patient, cost to healthcare system and significant variability/risk of artifacts [12]. Hence, several alternatives to urodynamics have been investigated over the past two decades such as urinary markers or ultrasonographic parameters [5]. To our knowledge this study is the first to explore CT texture analysis as a predictor of urodynamic findings in spina bifida patients or in any other neurologic patients. We found that some CT texture parameters were significantly associated with high risk urodynamic features such as poor bladder compliance suggesting that CT texture analysis of the bladder wall might be an alternative to urodynamics for spina bifida patients in selected situations.

Image texture analysis can be described as a wide range of techniques for quantification of gray-level patterns and pixel inter-relationships within an image. In other terms, it can be considered as a mathematical representation of image features that can be characterized in words. Texture analysis relies on a process that includes six steps: 1) images acquisition, 2) region of interest ROI definition, 3) ROI preprocessing, 4) feature extraction, 5) feature selection and 6) classification. However, none is specific and many parameters can vary in each step $[6-7,13]$. Therefore, collaborative efforts are mandatory to develop and validate protocols for each step. First, a global effort towards standardization of imaging is necessary. This effort requires standardization not only for image acquisition protocols, but also for volume of interest identification and features 
extractions. There is also a fundamental need that the medical and scientific communities design studies to enable the translation of imaging biomarkers from "bench" to clinical practice $[6-7,13]$. Though the use of retrospectively collected data is necessary to develop, test and evaluate texture analysis as an imaging biomarker, the risk to select significant features by chance and overfitting is major. Therefore, prospective studies to identify promising features from texture analysis with external validation in multicentric cohorts are necessary [13-14]. Finally, efforts should be directed towards the determination of the mechanisms beyond the correlations observed between clinical findings and corresponding texture features [13-14].

Our results suggest the potential usefulness of quantitative measures from texture analysis to detect detrusor overactivity or poor bladder compliance in spina bifida patients. We found skewness and kurtosis be statistically associated with the presence of poor bladder compliance and detrusor overactivity. These quantitative measures do not have simple qualitative description, but reflect subtle patterns of dependencies in the image [15]. Skewness is a measure of histogram asymmetry; a zero value indicates a symmetrical distribution around the mean. We speculate that in fibrotic, thickened poorly compliant bladders the resulting histograms from ROls are likely to be more skewed compared with normal compliance bladder with less heterogeneous cellular density [15]. The correlation between fibrosis and skewness that has been demonstrated in other conditions would support this idea of skewness indicating fibrosis responsible of decreased bladder compliance [16]. Kurtosis is another histogram-based method which measures histogram flatness. The increased kurtosis associated with detrusor overactivity could reflect the increase intensity variation in the bladder wall of these patients that might indicate mictrostructural changes [15].

One of the points of strength of our series was the systematic CT performed in all spina bifida patients at the first visit to the national referral center. While our findings suggest that CT texture analysis may provide valuable information regarding NLUTD, the exposure to ionizing radiation associated with the use of CT in this young patient population shall raise concerns [17-18] and prevent to advocate for the use of repeat CT as an alternative to repeat urodynamics. However, CT is commonly used in the 
neurourological management of spina bifida patients in the context of urinary tract infections or to detect urolithiasis [4, 19-20]. Abdominal CT are also frequently performed for other purposes in spina bifida patients, for instance for ventriculoperitoneal shunts issues [21]. Hence, rather that pleading for dedicated CT to replace urodynamics in some instance, the promise of CT texture analysis if confirmed might support the analysis of data from existing CT performed for other purposes to draw information regarding NLUTD. Such a tool might be of interest as part of multimodal neurourological follow-up protocols in spina bifida patients. Texture analysis can also be performed using magnetic resonance imaging (MRI). Future studies exploring the value or MRI feature parameters to predict high risk urodynamic features might open up another way to use radiomics as an alternative to urodynamics divested from the radiation exposure issue.

Our study has some limitations that should be acknowledged. First, it was a retrospective monocentric study, with a relatively small number of patients. Second, drawing of ROls was done manualiy, possibly introducing variability. Third, we performed two-dimensional texture analysis on regions of interest selected from three axial sections rather than three-dimensional analysis, which might allow a more precise evaluation of the picture heterogeneity and also improve reproducibility. However, several studies have shown that use of a single slice is sufficient for sampling and extracting subtle features relevant for clinical application [22]. Fourth, our results were obtained using open access texture analysis software and we cannot extend our conclusions to other software programs. The decision to select detrusor overactivity and poor bladder compliance as the relevant urodynamic outcomes of interest might be called into question as other urodynamic parameters such as Pdetmax or detrusor leak point pressure may have been regarded as valuable alternatives. The bladder condition at the time of the CT was not perfectly standardized which might be regarded as a shortcoming. However, the texture parameters were extracted from the bladder wall images and whether they might be influenced by the degree of bladder filling is unknown. We decided not to include a control group of healthy volunteers to maximize the chance that radiomic differences observed would reflect bladder function differences 
rather than differences inherent to the spina bifida condition itself. However, this methodological choice might be considered as a study's flaw. Another possible shortcoming of this study is that we did not include analysis of CT gross morphology data which have never been explored as possible predictors of urodynamic features and might therefore deserve further investigations. The fact that models that learn from data are developed from a single technique and a single classifier selected based on researchers' preference and experience could be regarded as a shortcoming of the radiomics study in general and of our study in particular. Finally, the number of covariates was large compared to sample size. We addressed this issue by using the LASSO penalised regression model, which is suitable for analysis of high dimensional data

\section{Conclusion}

Our findings demonstrate that CT texture analysis of the bladder wall might be an interesting tool to identify spina bifida patients with high risk urodynamic features. This suggests that CT texture analysis of the bladder wall might be a non-invasive alternative to urodynamic for spina bifida patients in selected clinical scenarios. Further investigations with new textural analysis and prospective validation of the textural candidates identified in this study and mandatory to develop an accurate and comprehensive multi-functional signature of high-pressure spina bifida bladder in the future. Assessment of the value of CT texture analysis in other neurologic conditions may also be of interest. 


\section{References}

1. Mitchell LE, Adzick NS, Melchionne J, Pasquariello PS, Sutton LN, Whitehead AS. Spina bifida. Lancet. 2004;364(9448):1885-95.

2. Gamé X, Grima F, Chartier-Kastler E, Ruffion A. Vesicosphincteric and sexual disorders associated with spina bifida and myelomeningocele. Prog Urol 2007;17(3):352-7.

3. Veenboer PW, Bosch JLHR, van Asbeck FWA, de Kort LMO. Upper and lower urinary tract outcomes in adult myelomeningocele patients: a systematic review. PloS One. 2012;7(10):e48399.

4. Blok B, Padilla-Fernández, Pannek J, D. et al. Neuro-Urology EAU Guidelines. In: Edn. presented at the EAU Annual Congress Copenhagen 2018.

5. Farag FF, Heesakkers JP. Non-invasive techniques in the diagnosis of bladder storage disorders. Neurourol Urodyn. 2011;30(8):1422-8.

6. Gillies RJ, Kinahan PE, Hricak H. Radiomics: Images Are More than Pictures, They Are Data. Radiology. 2016;278(2):563-77.

7. Yip SS, Aerts HJ. Applications and limitations of radiomics. Phys Med Biol. 2016 Jul 7;61(13):R150-66

8. Rosier PFWM, Schaefer W, Lose G, et al. International Continence Society Good Urodynamic Practices and Terms 2016: Urodynamics, uroflowmetry, cystometry, and pressure-flow study. Neurourol Urodyn. 2017; 36(5):1243-1260.

9. Gajewski JB, Schurch B, Hamid R, et al. An International Continence Society (ICS) report on the terminology for adult neurogenic lower urinary tract dysfunction (ANLUTD). Neurourol Urodyn. 2018; 37(3):1152-1161

10. Tarcan T, Sekerci CA, Akbal C, et al. Is $40 \mathrm{~cm} \mathrm{H}_{2} \mathrm{O}$ detrusor leak point pressure cutoff reliable for upper urinary tract protection in children with myelodysplasia? Neurourol Urodyn. 2017;36(3):759-763.

11. Seki N, Akazawa K, Senoh K, et al. An analysis of risk factors for upper urinary tract deterioration in patients with myelodysplasia. BJU Int. 1999 ;84(6):679-82. 
12. Veenboer PW, Bosch JL, Rosier PF, et al. Cross-sectional study of determinants of upper and lower urinary tract outcomes in adults with spinal dysraphism--new recommendations for urodynamic followup guidelines? J Urol. 2014;192(2):477-82.

13. Lubner MG, Smith AD, Sandrasegaran K, Sahani DV, Pickhardt PJ. CT Texture Analysis: Definitions, Applications, Biologic Correlates, and Challenges. Radiographics. 2017;37(5):1483-1503.

14. Chalkidou A, O'Doherty MJ, Marsden PK. False Discovery Rates in PET and CT Studies with Texture Features: A Systematic Review. PLoS One. 2015;10(5):e0124165.

15. Miles KA, Ganeshan B, Hayball MP. CT texture analysis using the filtrationhistogram method: what do the measurements mean? Cancer Imaging. 2013 $23 ; 13(3): 400-6$.

16. Lubner MG, Jones D, Kloke J, Said A, Pickhardt PJ. CT texture analysis of the liver for assessing hepatic fibrosis in patients with hepatitis C virus. $\mathrm{Br} J$ Radiol. 2018 in press. doi: 10.1259/bjr.20180153

17. Miglioretti DL, Johnson E, Williams A, et al. The use of computed tomography in pediatrics and the associated radiation exposure and estimated cancer risk. JAMA Pediatr. 2013 Aug 1;167(8):700-7.

18. Pearce MS, Salotti JA, Little MP, et al. Radiation exposure from CT scans in childhood and subsequent risk of leukaemia and brain tumours: a retrospective cohort study. Lancet. 2012;380(9840):499-505.

19. Santiago-Lastra $Y$, Cameron AP, Lai J, et al Urological Surveillance and Medical Complications in the United States Adult Spina Bifida Population.Urology. 2019;123:287292.

20. Duplisea JJ, Romao RL, MacLellan DL, Cox AR, Anderson PA. Urological Follow-up in Adult Spina Bifida Patients: Is There an Ideal Interval? Urology. 2016 ;97:269-272.

21. Solth A, Mukerji N, Strachan R. Reducing the radiation exposure from CT scanning in children with shunts: a nationwide survey and a departmental CT protocol. $\mathrm{Br} J$ Neurosurg. 2018;32(5):558-562.

22. Lubner MG, Stabo N, Lubner SJ, et al. CT textural analysis of hepatic metastatic colorectal cancer: pre-treatment tumor heterogeneity correlates with pathology and clinical outcomes. Abdom Imaging. 2015;40(7):2331-7. 
Figure legends

Figure 1: Illustration of lesion delineation

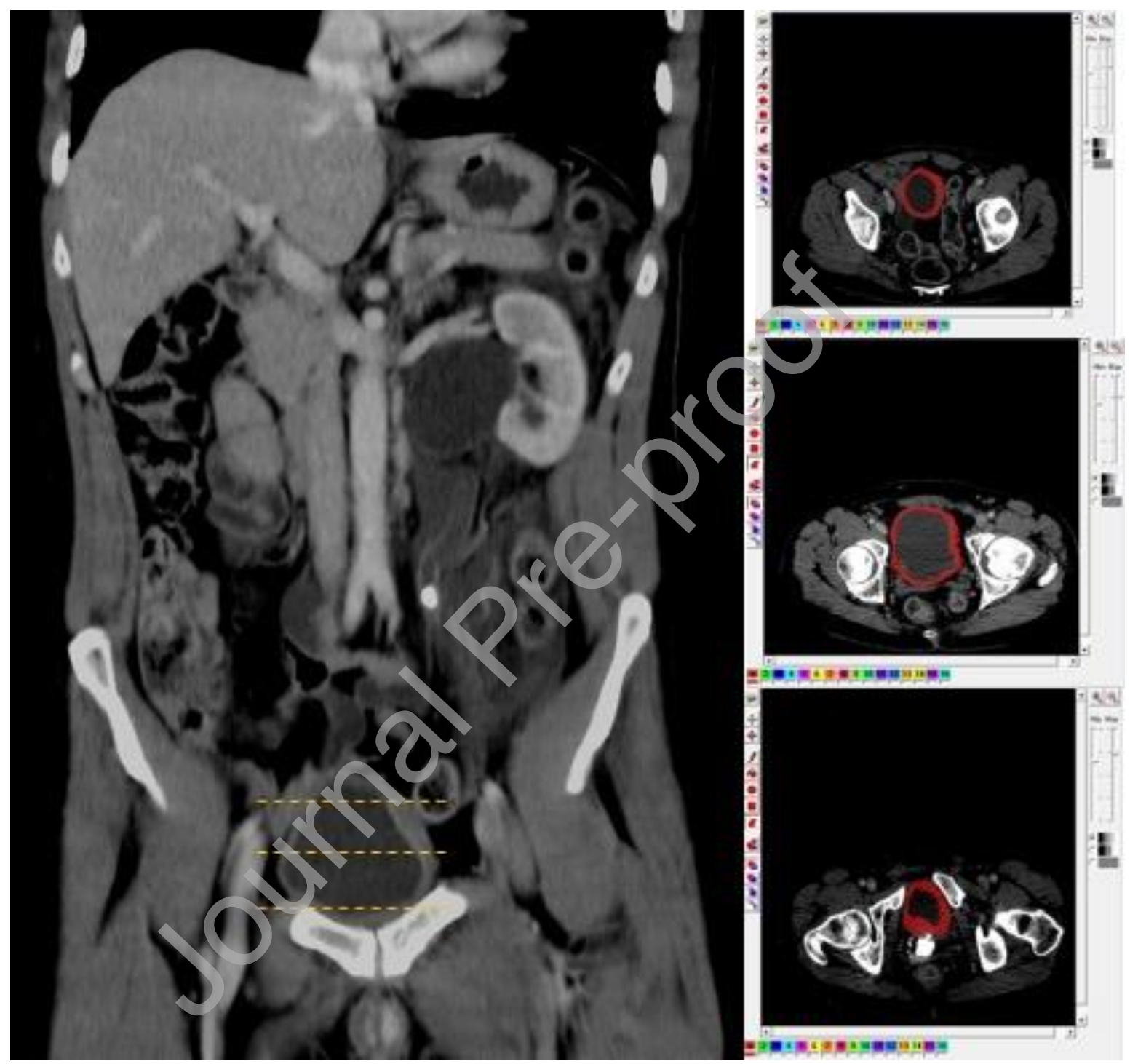


Figure 2: Overview workflow of the method

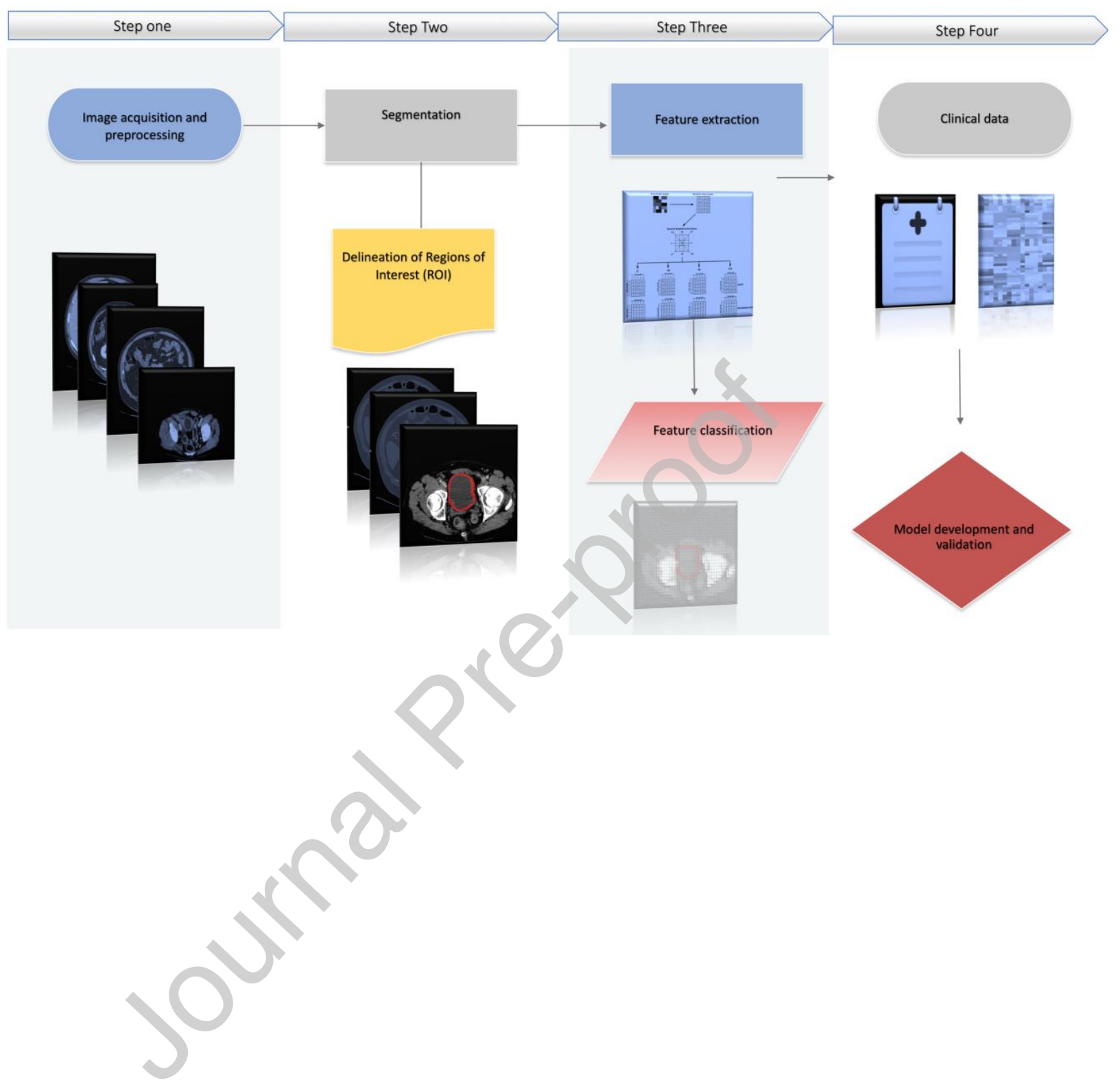


Table 1: Multivariate Logistic proportional hazards regression analyses of texture features and clinical parameters selected by Lasso penalized logistic regression analysis for predicting poor bladder compliance

\begin{tabular}{|c|c|c|}
\hline & $\begin{array}{c}\text { Odds-ratio } \\
{[\text { Cl-95\%] }}\end{array}$ & p-value \\
\hline Age & $\begin{array}{c}0.96 \\
{[0.92-0.99]}\end{array}$ & 0.2 \\
\hline Type of Spinal dysraphism & $\begin{array}{c}1.29 \\
{[0.29-5.78]}\end{array}$ & 0.73 \\
\hline Skewness & 0.41 & 0.04 \\
\hline S11sumVarnc & {$[0.15-0.98]$} & 0.245 \\
\hline
\end{tabular}


Table 2: Multivariate Logistic proportional hazards regression analyses of texture features and clinical parameters selected by Lasso penalized logistic regression analysis for predicting detrusor overactivity

\begin{tabular}{|c|c|c|}
\hline & $\begin{array}{c}\text { Odds-ratio } \\
{[\text { Cl-95\%] }}\end{array}$ & p-value \\
\hline Age & $\begin{array}{c}1.01 \\
{[0.96-1.06]}\end{array}$ & 0.71 \\
\hline Male gender & $\begin{array}{c}1.16 \\
{[0.27-5.00]}\end{array}$ & 0.85 \\
\hline Kurtosis & 1.12 & 0.026 \\
\hline Type of spinal dysraphism & {$[1.01-1.55]$} & 0.17 \\
\hline
\end{tabular}

